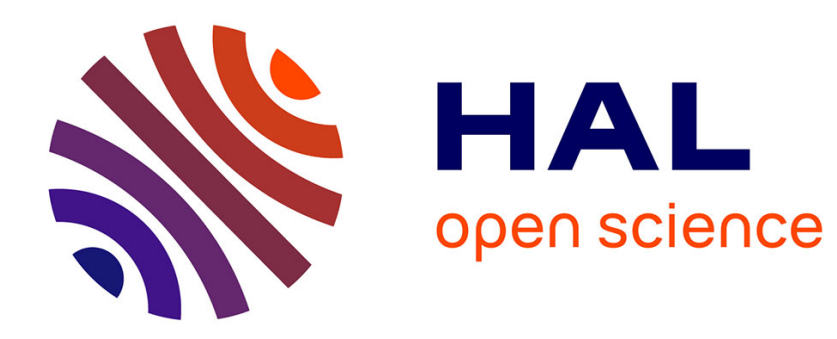

\title{
Perturbation de la lueur négative d'une décharge luminescente par une onde hyperfréquence
}

\author{
J. Bloyet
}

\section{To cite this version:}

J. Bloyet. Perturbation de la lueur négative d'une décharge luminescente par une onde hyperfréquence. Revue de Physique Appliquée, 1974, 9 (6), pp.981-987. 10.1051/rphysap:0197400906098100 . jpa00243869

\section{HAL Id: jpa-00243869 https://hal.science/jpa-00243869}

Submitted on 1 Jan 1974

HAL is a multi-disciplinary open access archive for the deposit and dissemination of scientific research documents, whether they are published or not. The documents may come from teaching and research institutions in France or abroad, or from public or private research centers.
L'archive ouverte pluridisciplinaire HAL, est destinée au dépôt et à la diffusion de documents scientifiques de niveau recherche, publiés ou non, émanant des établissements d'enseignement et de recherche français ou étrangers, des laboratoires publics ou privés. 
Classification

Physics Abstracts

6.700

\title{
PERTURBATION DE LA LUEUR NÉGATIVE D’UNE DÉCHARGE LUMINESCENTE PAR UNE ONDE HYPERFRÉQUENCE
}

\author{
J. BLOYET
}

Laboratoire de Physique des Plasmas (*)

Université de Paris-Sud, Centre d'Orsay 91405 Orsay, France

(Reçu le 3 janvier 1974, révisé le 5 juin 1974)

\begin{abstract}
Résumé. - Sous l'influence d'une onde électromagnétique, l'énergie thermique des électrons d'une décharge augmente. Cet accroissement d'énergie peut modifier certains processus microscopiques de la décharge et peut ainsi entraîner une variation du courant de la décharge.

Le présent article est l'étude des mécanismes de la perturbation provoquée par une onde hyperfréquence dans la lueur négative d'une décharge d'argon.
\end{abstract}

\begin{abstract}
An applied electromagnetic wave will increase the thermal energy of the electrons of a gaseous discharge. This energy increase can modify certain microscopic processes in the discharge, and thereby modify the discharge current. We discuss in detail the negative glow of an argon discharge illuminated by a high frequency signal.
\end{abstract}

1. Introduction. - La modification de la caractéristique volt-ampère d'une décharge luminescente, soumise à l'influence d'une onde électromagnétique haute fréquence, a fait l'objet de nombreuses recherches ayant pour but la réalisation d'un détecteur d'ondes hyperfréquences.

Le travail que nous présentons a pour objet de rechercher dans quelles conditions on obtient le maximum de variation du courant de la décharge et de préciser les mécanismes de cette variation dans une décharge d'argon, celle-ci étant soumise à l'influence d'une onde électromagnétique d'une puissance de quelques $\mathrm{mW}$.

2. Description du montage expérimental et des circuits de mesure. - 2.1 Montage EXPéRIMENTAL. Nous donnons un schéma de principe du montage utilisé (Fig. 1).

La décharge est établie dans un tube en pyrex $(\varnothing 8 \mathrm{~mm})$ entre deux électrodes planes en fer $(\varnothing 7,5 \mathrm{~mm})$.

Les ondes hyperfréquences $(10 \mathrm{GHz})$ sont dirigées par un guide rectangulaire. Le banc se compose de deux circuits, l'un provoque la perturbation, l'autre permet les mesures (Fig. 2).

2.2 DispositiF D'INTERACTION ONDE-PLASMA. Hormis la colonne positive, les différentes régions caractéristiques d'une décharge luminescente ont de

(*) Laboratoire Associé au C. N. R.S.

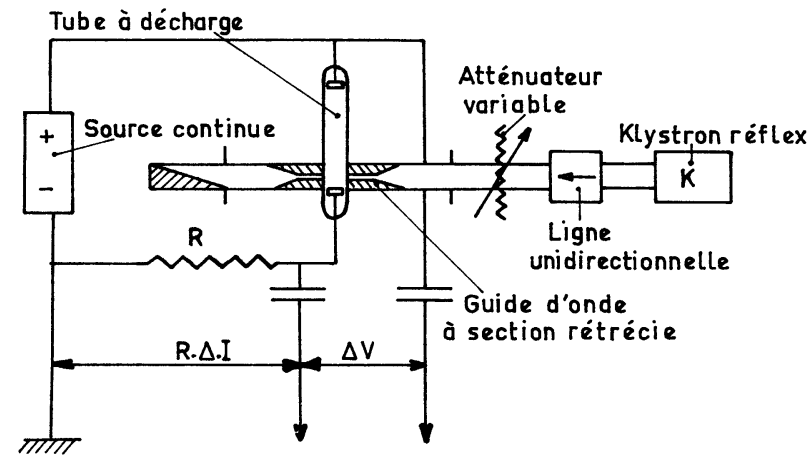

FIG. 1. - Schéma de principe.

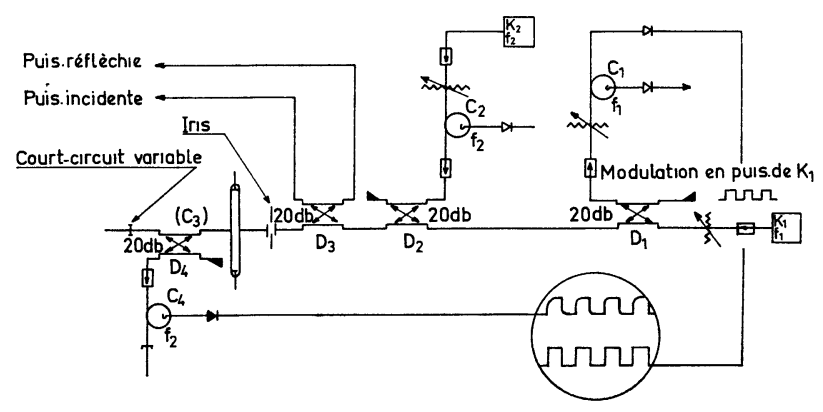

FIG. 2. - Montage utilisé pour observer les variations de $N_{\mathrm{e}}$.

petites dimensions longitudinales (quelques $\mathrm{mm}$ à quelques $\mathrm{cm}$ ). Pour pouvoir procéder à une analyse aussi fine que possible des processus de la perturbation des différentes régions de la décharge, il est nécessaire d'utiliser un faisceau d'ondes ayant une ouverture 
angulaire petite. Un moyen simple d'y parvenir est de faire traverser le guide par le tube à décharge luimême en diminuant la section du guide pour obtenir la meilleure résolution spatiale (Fig. 1).

2.3 Circuits DE MESURE. - Le tube à décharge est disposé perpendiculairement au grand côté du guide d'onde qu'il traverse en son plan médian. La puissance $\mathrm{H}$. F. provoquant la perturbation $(0-0,5 \mathrm{~W})$ est délivrée par un klystron $\mathrm{K}_{1}$ et modulée en signaux carrés à $1000 \mathrm{~Hz}$ (Fig. 2).

Pour la mesure de la densité électronique, on forme une cavité de mesure $\mathrm{C}_{3}$ en plaçant, d'une part, un court-circuit variable à l'extrémité du banc d'autre part, un iris entre le coupleur $\mathrm{D}_{3}$ et le guide à section rétrécie. Les mesures du changement $\Delta f$ de la fréquence de résonance lorsque l'on fait varier le courant de la décharge, permettent, en principe, de calculer la densité électronique moyenne de celle-ci.

L'observation de la variation du flux lumineux dans la région soumise à l'influence de l'onde se fait en plaçant une photo-diode face à un orifice percé dans le guide d'onde de sorte que la visée coïncide avec l'intersection de l'axe longitudinal du guide d'onde et l'axe du tube à décharge.

L'onde H. F. provoque une variation du courant de la décharge à une fréquence égale à la fréquence de modulation de l'onde perturbatrice. Cette variation du courant provoque une variation de potentiel aux bornes de la résistance de charge $R$. Ce signal, que nous appelons signal détecté, est capté à travers une
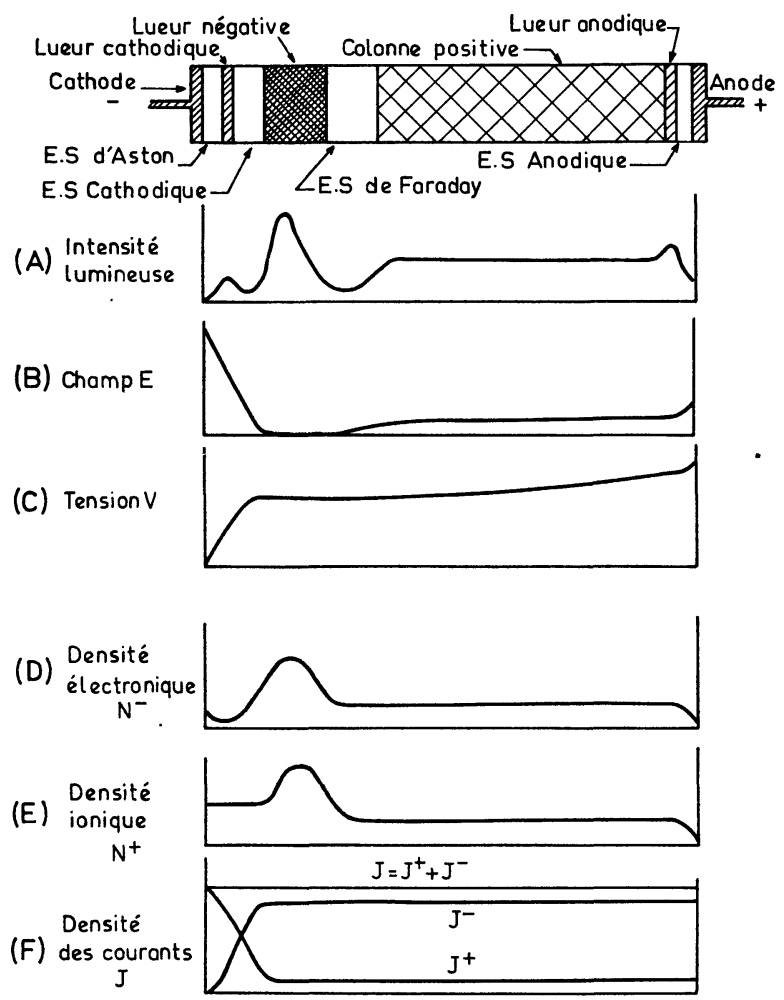

FIG. 3. - Caractéristiques longitudinales d'une décharge luminescente. capacité et appliqué sur l'amplificateur vertical d'un oscilloscope.

2.4 CholX DES CONDITIONS DE TRAVAIL. - Les premières observations montrent que la perturbation provoquée par l'onde hyperfréquence existe quelle que soit l'orientation du champ de l'onde par rapport au champ continu de la décharge; son amplitude dépend :

- de la région de la décharge soumise à l'onde électromagnétique (Fig. 4);

- des conditions de fonctionnement de la décharge, pression du gaz (Fig. 5), courant de la décharge (Fig. 6) ;

- de la puissance de l'onde électromagnétique (Fig. 7).

Ces résultats permettent de tirer quelques conclusions. L'amplitude du signal détecté croît avec la

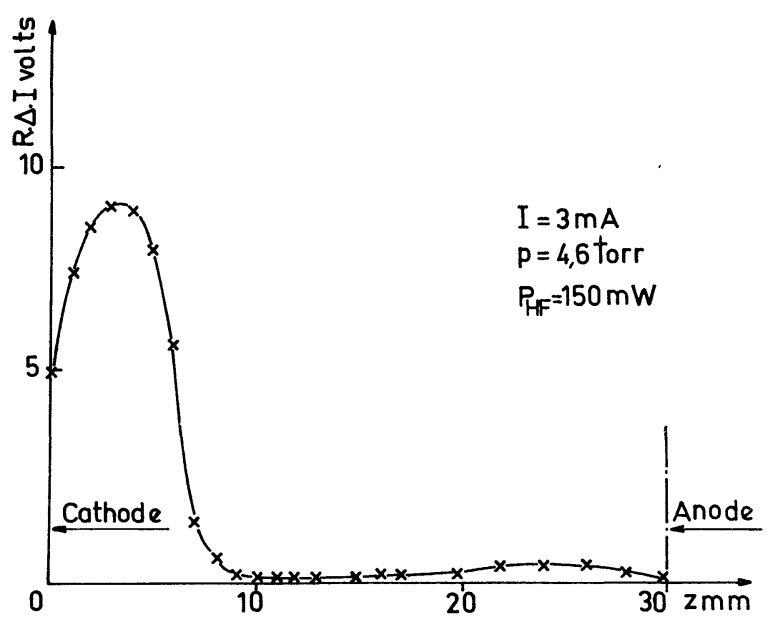

Fig. 4. - Amplitude du signal détecté en fonction de $Z$.

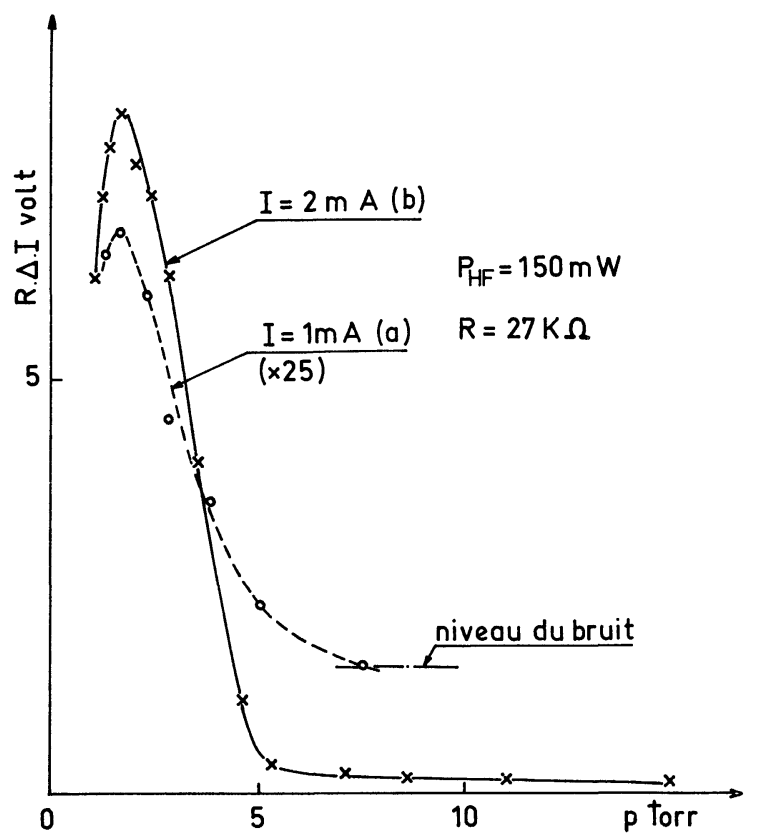

Fig. 5. - Amplitude de la détection en fonction de la pression. 


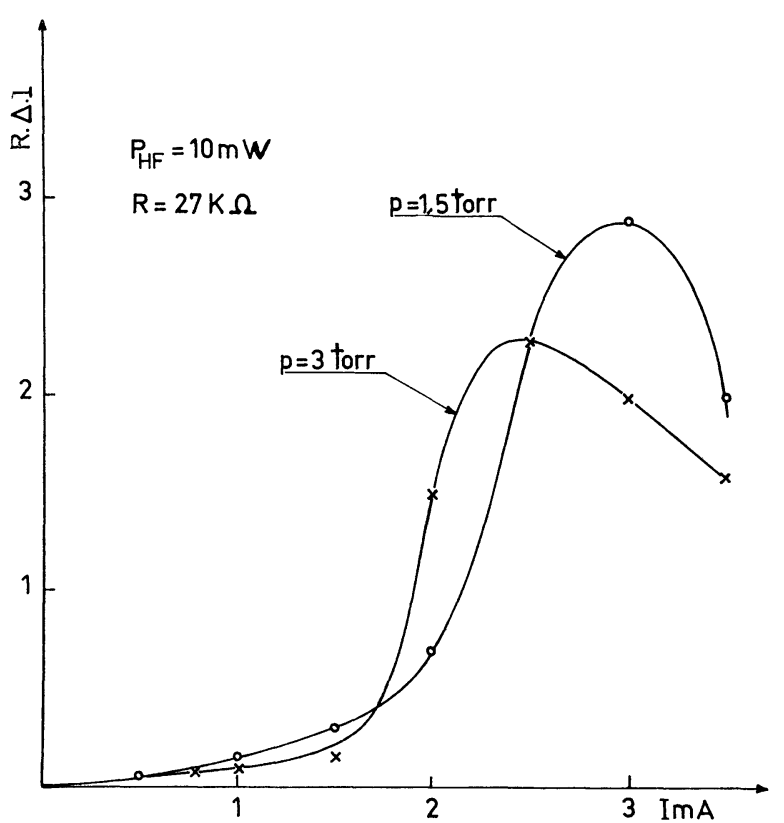

Fig. 6. - Signal détecté en fonction du courant de la décharge.

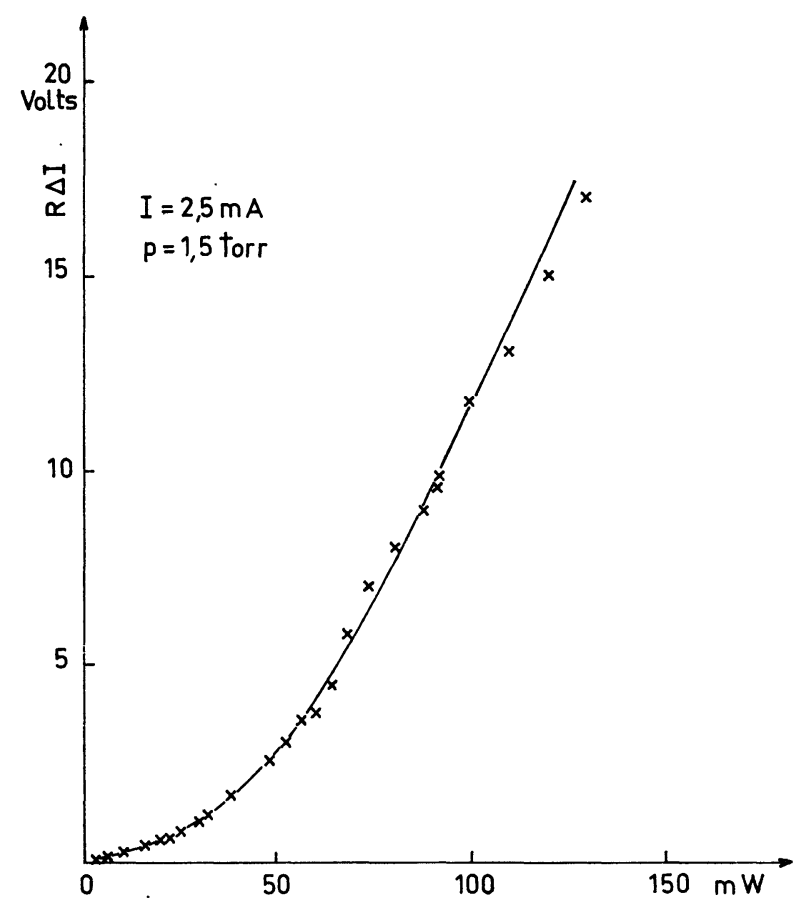

Fig. 7. - Amplitude du signal détecté en fonction de la puissance H. F. incidente.

puissance $\mathrm{H}$. F. La région la plus sensible est la lueur négative et l'on obtient un signal maximum pour un courant de décharge de $3 \mathrm{~mA}$ et une pression de 1,5 torr. Ces conditions correspondent à un fonctionnement de la décharge en régime anormal (Fig. 8).

2.5 ETUDE DES CONDITIONS DE FONCTIONNEMENT DE LA RÉGION CATHODIQUE D'UNE DÉCHARGE LUMINESCENTE EN RÉGIME ANORMAL. - Pour interpréter l'effet des ondes électromagnétiques sur la lueur négative il est nécessaire d'en connaître qualitativement et quantitativement ses caractéristiques. Nous allons

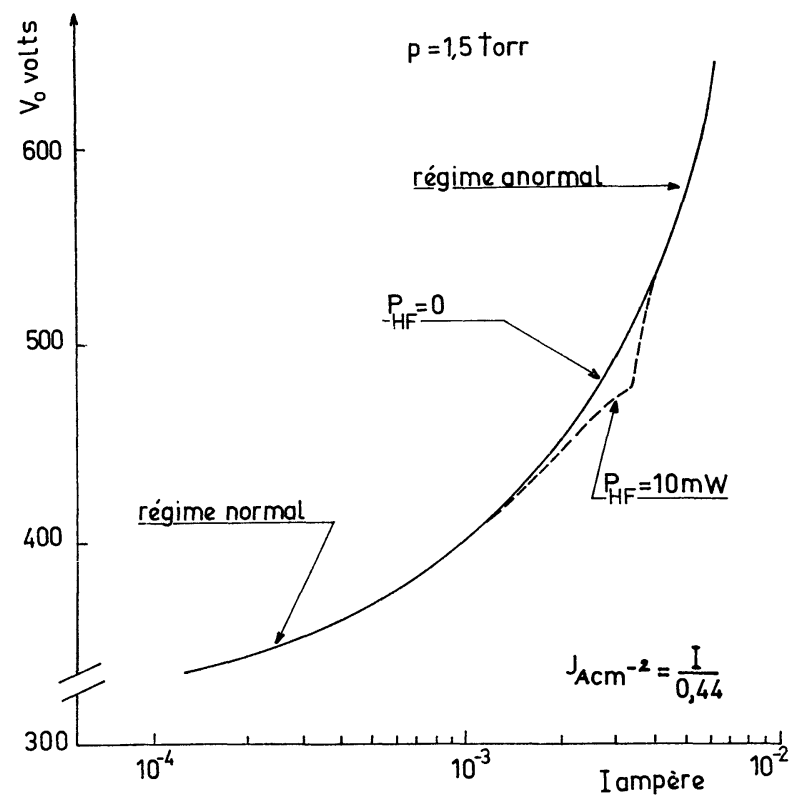

FIG. 8. - Caractéristique V. A.

succinctement donner quelques caractéristiques $\mathrm{du}$ fonctionnement de la région cathodique d'une décharge luminescente fonctionnant en régime anormal.

$\mathrm{Du}$ fait du fonctionnement même de la décharge il y a accumulation d'ions positifs au voisinage de la cathode ; il en résulte, dans cette région, un champ électrique $E$ important qui absorbe la majeure partie de la différence de potentiel appliquée aux bornes du tube. Les mesures de $E$ montrent que ce champ décroît quasi linéairement de la cathode vers la lisière de la lueur négative où il est à peu près nul (Fig. 3).

Si l'on rapproche suffisamment les électrodes on peut limiter la décharge luminescente à la région cathodique (chute cathodique et lueur négative); le bruit est alors considérablement diminué et la différence de potentiel mesurée entre les électrodes est peu différente de la tension absorbée dans la chute cathodique, ce dont nous ferons usage par la suite.

La neutralisation des ions sur la cathode provoque l'émission électronique. En régime anormal, elle est essentiellement due aux ions positifs provenant de la lueur négative [1].

La lueur négative est caractérisée par une densité de charge élevée et un champ électrique très faible ; les électrons accélérés dans la chute cathodique (généralement appelés électrons du $1^{\text {er }}$ groupe), pénètrent dans la lueur négative, y produisent une ionisation importante et s'y thermalisent. Les électrons secondaires ainsi formés (électrons du $2^{\mathrm{e}}$ groupe) possèdent eux-mêmes, en moyenne, une énergie suffisante pour ioniser et exciter le gaz. Il en résulte la création d'un $3^{e}$ groupe d'électrons de densité élevée $\left(10^{10}\right.$ à $\left.10^{12} \mathrm{~cm}^{-3}\right)$ dont la fonction de distribution est approximativement maxwellienne, la température correspondante étant faible $(0,1 \mathrm{eV}$ à $1 \mathrm{eV})$; le plasma qu'ils forment avec les ions corres- 
pondants est à peu près équi-potentiel. Contrairement à ce qui se produit dans la chute cathodique, les pertes de particules chargées dans la lueur négátive ne sont pas négligeables; ces pertes sont dues à la diffusion ambipolaire radiale des particules, à leur diffusion axiale et à leur recombinaison en volume.

Dans notre cas, la recombinaison en volume domine et plus précisément celle du type dissociatif (Manus, C., Communication privée, Service de Physique Appliquée, C.E.N.-Saclay), pour des pressions comprises entre 0,1 et quelques torr.

2.6 EXPRESSION DU COURANT DE LA DÉCHARGE EN FONCTION DE LA TEMPÉRATURE ÉLECTRONIQUE DANS LA LUEUR NÉGATIVE. - La densité du courant de la décharge s'exprime au niveau de la cathode par

$$
I=I_{0}^{+}+I_{0}^{-}=(1+\gamma) I_{0}^{+}
$$

où $\gamma$ est le second coefficient de Townsend.

En régime anormal on a $I_{0}^{+} \simeq q \Phi_{d}^{+}$, où $\Phi_{d}^{+}$le flux des ions à la lisière cathodique de la lueur négative est donné par $\Phi_{d}^{+}=-D_{\mathrm{a}} \nabla N^{+}$avec $\left(\nabla N^{+} \simeq N_{\mathrm{LN}}^{+} / l\right)$.

Dans notre cas, nous savons que les pertes par recombinaison dissociatives sont prépondérantes, on a à l'équilibre

$$
n_{\mathrm{L}}=\alpha_{\mathrm{r}} N_{\mathrm{LN}}^{2}
$$

le taux de l'ionisation $n_{\mathrm{L}}$, dûe au courant $I_{0}^{-} \mathrm{e}^{\bar{\alpha}^{\alpha} d}$ sortant de la chute cathodique est

$$
n_{\mathrm{L}}=I_{0}^{-} \frac{\mathrm{e}^{\bar{\alpha} \cdot d}}{q \lambda_{\mathrm{i}}}=\frac{\gamma}{\lambda_{\mathrm{i}}} \Phi_{0}^{+} \mathrm{e}^{\bar{\alpha} \cdot d}
$$

où $\Phi_{0}^{+}$est le flux d'ions bombardant la cathode, $\lambda_{\mathbf{i}}$ le libre parcours moyen d'ionisation, $\bar{\alpha}$ la valeur moyenne du premier coefficient de Townsend.

Le coefficient $\alpha_{r}$ de recombinaison dissociatif est relié à la température électronique $T_{\mathrm{e}}$ par l'expression [2, 3]

$$
\alpha_{\mathrm{r}}=\alpha_{\mathrm{r} 0}\left(\frac{300}{T_{\mathrm{e}}}\right)^{b}
$$

où $b$ est un coefficient dépendant de la nature du gaz.

On en déduit que la densité ionique moyenne, dans la lueur négative

$$
N_{\mathrm{LN}}^{+}=\left[\frac{\gamma \Phi_{0}^{+} \mathrm{e}^{\bar{\alpha} . d}}{\lambda_{\mathrm{i}} \alpha_{\mathrm{r} 0}}\left(\frac{T_{\mathrm{e}}}{300}\right)^{b}\right]^{1 / 2} .
$$

On sait, d'une part [5] que

$$
\Phi_{0}^{+}=2 \varepsilon_{0} \sqrt{\frac{\lambda_{t}}{M}}\left(\frac{V_{0}^{3 / 2}}{d^{5 / 2}}\right)
$$

avec $\lambda_{t}$ libre parcours moyen de transfert de charge des ions, $V_{0}$ le potentiel de la chute cathodique, $d$ la longueur de la chute cathodique et que, d'autre part, le coefficient de diffusion ambipolaire est donné par l'expression

$$
D_{a}=\frac{k\left(T_{\mathrm{i}}+T_{\mathrm{e}}\right)}{M v_{\mathrm{in}}}
$$

où $v_{\text {in }}$ est la fréquence de collision ion-neutre et $T_{\mathrm{i}}$ la température des ions.

Des équations ci-dessus, on déduit la relation entre le courant de la décharge et la température électronique dans la lueur négative

$$
I=A \sqrt{\frac{V_{0}^{3 / 2}}{d^{5 / 2}}}\left(\frac{T_{\mathrm{i}}}{T_{\mathrm{e}}}+1\right) T_{\mathrm{e}}^{(1+b / 2)}
$$

où $A$ est un coefficient dépendant de la fréquence de collision ion-neutre, du libre parcours moyen d'ionisation, du libre parcours moyen de transfert de charge des ions et du premier coefficient de Townsend.

On remarque que, toutes grandeurs égales par ailleurs, le courant de la décharge dépend directement de la température électronique dans la lueur négative et que dans le cas de l'Argon où $b=2 / 3$, on a

$$
I=B\left(\frac{T_{\mathrm{i}}}{T_{\mathrm{e}}}+1\right) T_{\mathrm{e}}^{4 / 3}
$$

Dans $[4,5]$ on montre comment l'irradiation de la lueur négative d'une décharge luminescente, à l'aide d'une onde hyperfréquence, provoque une élévation de la température électronique ; il est donc clair que l'élévation de la température électronique entraîne une augmentation du courant de la décharge.

Pour les conditions de fonctionnement suivantes : $V_{0}=5 \times 10^{2} \mathrm{~V}, I=3 \times 10^{-3} \mathrm{~A}, \quad p=1$ torr, nous avons mesuré une variation de tension aux bornes d'une résistance de charge de $30 \mathrm{~K} \Omega$, égale à $3 \mathrm{~V}$, pour une puissance $H$. F. absorbée égale à $1 \mathrm{~mW}$ (ce qui correspond à une puissance incidente voisine de $10 \mathrm{~mW}$ ).

Dans ces conditions, nous avons calculé

$$
\Delta T_{\mathrm{e}} / T_{\mathrm{e}} \sim 0,25
$$

Cette variation de température est importante par rapport à la température initiale. Il est intéressant de constater que, dans notre cas, cette variation de $T_{e}$ entraîne une variation du coefficient de recombinaison dissociatif de l'ordre de $15 \%$ (cf. [2]).

Remarque. - Cette importante variation de la température électronique provoque aussi une variation de la fréquence de collision électron-neutre $v_{\text {en }}$ [6] qui, dans l'argon, à cause du seuil Ramsauer, peut entraîner une variation du courant de la décharge. On montre [7] que dans les conditions ci-dessus $\log v_{\mathrm{ee}} / \beta v_{\mathrm{en}} \sim 5$ (où $v_{\mathrm{ee}}$ est la fréquence de collision électron-électron et $\beta=2 m / M$ ) ce qui signifie que la répartition des vitesses électroniques se fait principalement par collision électron-électron. Cette remarque se trouve confirmée par les travaux de Kantorek [8] qui obtient des résultats identiques dans l'hélium et le néon où le seuil Ramsauer est inexistant. 
3. Résultats expérimentaux. - 3.1 Evolutions SIMULTANÉES DE $\Delta \mathrm{I}, N_{\mathrm{L}}^{-}, \Phi$, EN FONCTION DU COURANT DE DÉCHARGE POUR UNE PUISSANCE H. F. INCIDENTE FIXE. - La figure 9 indique que l'intensité du flux lumineux $\Phi$ varie à peu près linéairement avec le courant de décharge ; il en est de même pour la densité électronique, ce qui est conforme aux résultats obtenus par Stern [9] et Cellarius et al. [11]. La variation de $\Delta I$ est positive et son amplitude passe par un maximum ; ceci est en accord avec les résultats obtenus par Kantorek [8] et Séverin [11].

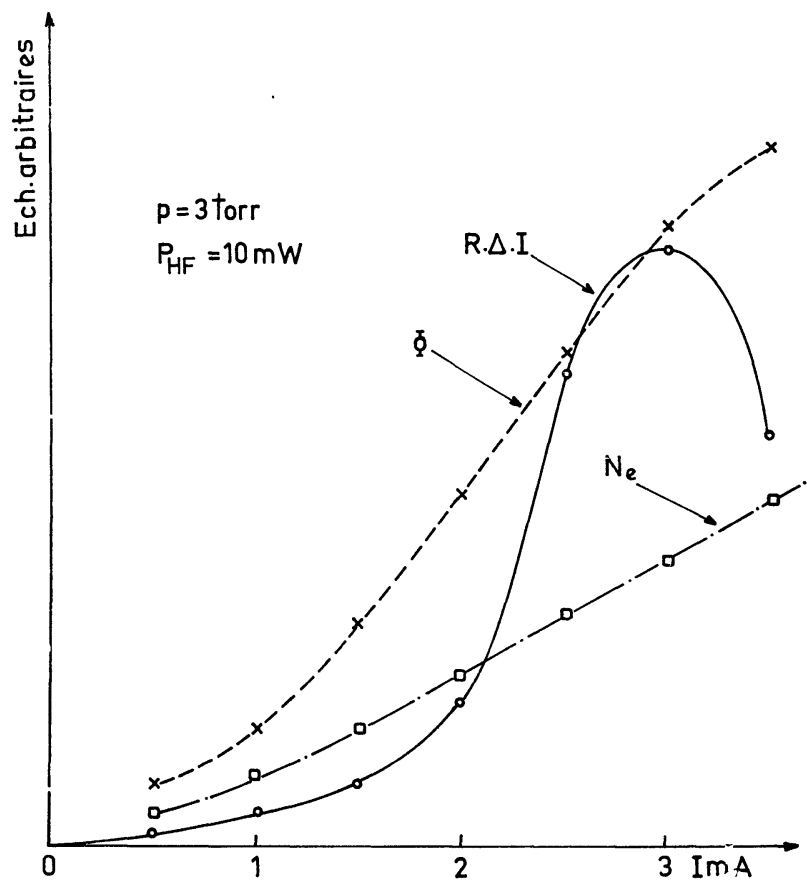

FIG. 9. - Variation de $N \overline{\mathrm{L}}, \Phi$ et $\Delta I$ en fonction de $I$.

La variation quasi linéaire de $N_{\mathrm{L}}^{-}$avec $I$ démontre que la vitesse de dérive des électrons du $3^{e}$ groupe de la lueur ne varie pas quand $J$ varie.

Pour expliquer l'allure de variation de $\Phi$ avec $I$, il faut connaître l'origine du flux lumineux dans la lueur. Certains auteurs pensent qu'il est dû à l'excitation du gaz par les électrons du $2^{\mathrm{e}}$ groupe, tandis que d'autres l'attribuent aux radiations émises lors des recombinaisons des ions avec les électrons du $3^{\mathrm{e}}$ groupe. Il est vraisemblablement dû aux deux processus comme l'indique aussi Badareu [12]. La lumière émise par le deuxième processus est sûrement très importante dans certaines conditions de fonctionnement. Deux expériences le confirment :

1) Badareu cite des expériences dans lesquelles on chauffe localement les électrons de la lueur négative en appliquant une tension entre deux grilles : on y constate une diminution de la lumière émise.

2) Dans notre cas, nous constatons que l'application de puissance $H$. F. sur la lueur négative a pour effet de diminuer la lumière émise. Rappelons que le coefficient de recombinaison varie comme $T_{\mathrm{e}}^{-2 / 3}$.

3.2 Evolutions SimultanéES DE $\Delta I, N_{\mathrm{L}}^{-}, \Phi$ eN FONCTION DE LA POSITION DU TUBE DANS LE GUIDE D'ONDE. - La figure 10 montre que $\Delta I, N_{\mathrm{L}}^{-}$et $\Phi$ passent par des maxima situés près de la cathode et qui ne coïncident généralement pas entre eux. Ces mesures sont en accord avec celles de [9] et [13]

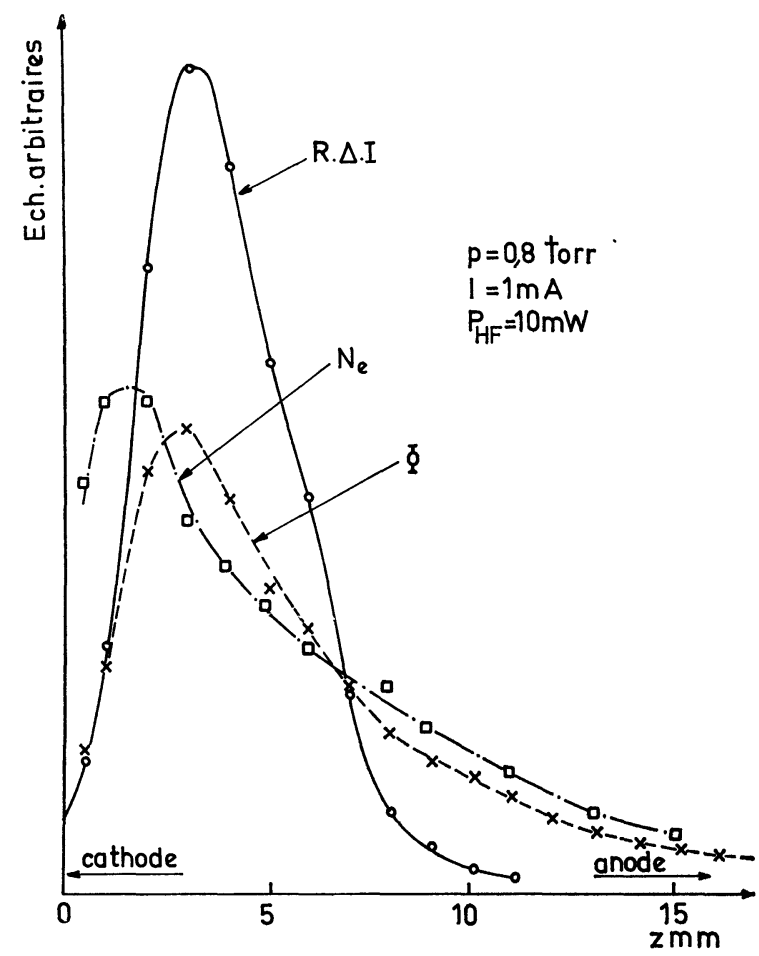

FIg. 10. - Variation de $N_{\overline{\mathrm{L}}}^{\bar{S}} \Phi$ et $\Delta I$ en fonction de $Z$.

D'après ces auteurs, le lieu du maximum de luminosité correspond au lieu du minimum de température des électrons du $3^{\mathrm{e}}$ groupe.

$\mathrm{Ce}$ résultat joue en faveur du $2^{\mathrm{e}}$ processus décrit précédemment.

3.3 RÉPONSE DE $N_{\mathrm{L}}^{-}, \Phi, I$, A UNE IMPULSION DE PUISSANCE H. F. - L'impulsion de puissance H. F. entraîne une augmentation de $N_{\mathrm{L}}^{-}$et de $I$ et une diminution de $\Phi$ (cf. fig. 11).

Ces résultats sont en accord avec l'hypothèse d'un processus de pertes par recombinaison :

- l'augmentation de la température due à la puissance H.F. diminue le coefficient de recombinaison et augmente la densité électronique (et ionique) ;

- l'augmentation des densités ionique et électronique contribue à celle du courant.

Quant à la diminution du coefficient de recombinaison, il contribue à la diminution de l'intensité lumineuse.

Nous constatons que les temps de réponse, montée 


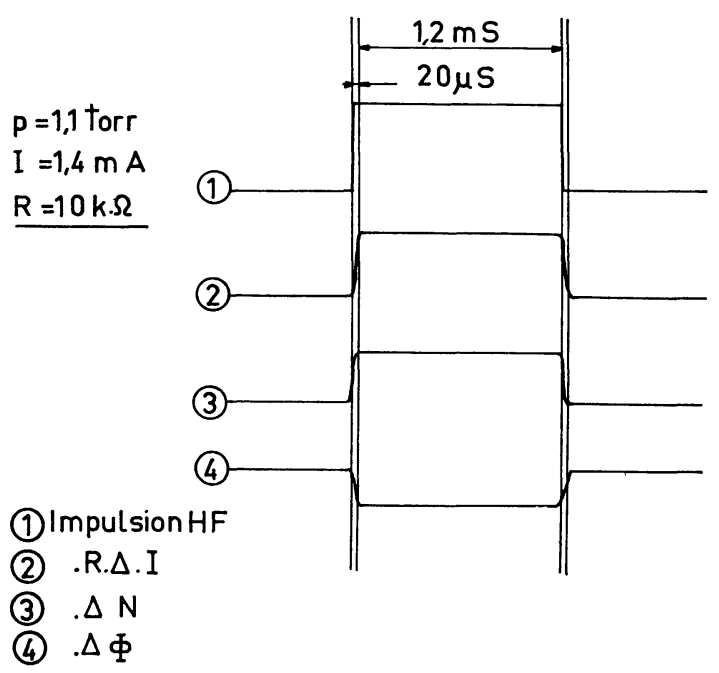

Fig. 11. - Variation de $N_{\mathrm{L}}, \Phi$ et $\Delta I$ en fonction de $t$.

et descente, sont sensiblement les mêmes, ce qui signifie que le même processus est responsable de l'augmentation et de la diminution de la densité. La mesure du temps de montée $\tau$, pour différents courants ou différentes pressions, montre que $\tau$ est de l'ordre de 10-20 $\mu$ s. Cette valeur est tout à fait comparable au temps de recombinaison $\tau_{\mathrm{r}} \sim 1 / \alpha_{\mathrm{r}} N_{\mathrm{L}}^{-}$

\section{$2,5 \mu \mathrm{s} \lesssim \tau_{\mathrm{r}} \lesssim 2,5 \mu \mathrm{s}$}

$$
\text { pour } 10^{18} \mathrm{~m}^{-3} \gtrsim N_{\mathrm{L}}^{-} \gtrsim 10^{17} \mathrm{~m}^{-3} \text { et } T_{\mathrm{e}} \sim 0,1 \mathrm{eV} \text {. }
$$

Nota. - Il est à noter que les paramètres de ce type de décharge fluctuent dans le temps. L'important, cependant, est la forme des courbes, c'est-à-dire la dépendance fonctionnelle des paramètres.

4. Mécanisme de pertes de particules dans la lueur négative. - Afin de préciser le processus de pertes de charges dans les conditions de travail de notre expérience, nous avons étudié le temps de relaxation de la variation de la densité électronique dans la postdécharge. Nous savons que les processus de pertes de particules chargées, dans une décharge luminescente, sont dus aux pertes par recombinaison et aux pertes par diffusion. Si le processus de pertes par recombinaison est prédominant en fonctionnement continu, il peut le rester durant un court temps après la coupure du circuit d'alimentation pour ensuite devenir négligeable devant le processus de diffusion. Si tel est le mécanisme de la post-décharge, on peut écrire :

$$
\frac{\mathrm{d} N^{-}}{\mathrm{d} t}=-\alpha_{\mathrm{r}} N^{-2}-\frac{D_{a}}{\Lambda^{2}} N^{-}
$$

et, dans notre cas, où les pertes par recombinaison sont plus grandes que les pertes par diffusion (cf. Appendice 8 dans [4]) on en déduit :

$$
\frac{N_{\mathrm{e} 0}}{N_{\mathrm{e}}}=1+N_{\mathrm{e} 0} \alpha_{\mathrm{r}} t
$$

Les résultats de la figure 12 nous indiquent, pour $p=10$ torr et pendant toute la durée de la mesure (300 $\mu \mathrm{s}$ ) et pour $p=2,4$ torr et pour $t<120 \mu \mathrm{s}$, que le mécanisme de perte par recombinaison contrôle la post-décharge.

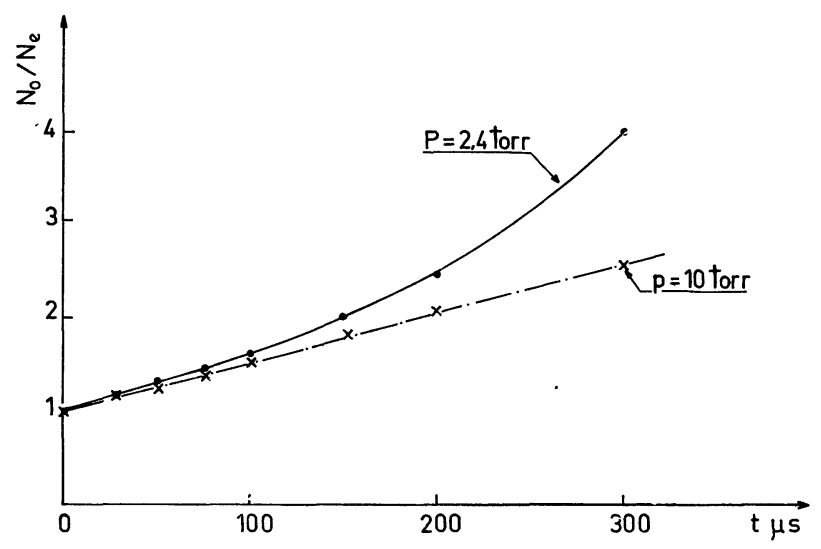

Fig. 12. - Evolution de $N_{0} / N_{\mathrm{e}}$ en fonction du temps dans la post-décharge.

5. Conclusion. - Nous avons montré que la lueur négative d'une décharge luminescente en régime anormal est une région très sensible aux ondes hyperfréquences. La forte densité d'un groupe d'électrons et sa faible température favorisent les pertes par recombinaison. Du fait qu'en régime anormal l'entretien de la décharge est contrôlé essentiellement par les ions de la lueur négative, on peut décrire le mécanisme de la perturbation dans la lueur négative de la manière suivante : sous l'effet de l'onde hyperfréquence les électrons acquièrent de l'énergie thermique provoquant une diminution du coefficient de recombinaison, donc une augmentation de la densité des charges. Cette dernière entraîne une augmentation directe du courant de la décharge par augmentation du courant de dérive et une augmentation indirecte par accroissement de l'émission secondaire cathodique consécutive à l'augmentation du nombre d'ions diffusant de la lueur négative vers la chute cathodique.

On peut espérer obtenir une plus grande variation du courant en utilisant, comme atmosphère, un gaz possédant un coefficient de recombinaison plus sensible aux variations de la température électronique. On peut aussi imaginer un montage permettant un meilleur couplage entre l'onde et la décharge.

Les résultats obtenus par certains auteurs montrent qu'il est possible d'obtenir un signal négatif $(-\Delta I)$ (dans certaines conditions de fonctionnement, nous avons fait la même observation au niveau de l'espace sombre de Faraday). Les conditions de fonctionnement particulières à ces expériences suggèrent que le mécanisme de la perturbation ait, pour origine, une diminution de la densité des charges dans la zone irradiée, par suite de l'augmentation des pertes par 
diffusion radiale. Du fait d'une faible densité des charges et d'une température électronique de quelques eV, l'espace sombre de Faraday se trouve être une région propice pour que les pertes par diffusion soient prépondérantes devant les pertes par recombinaison. Une expérience réalisée pour étudier particulièrement ce type de perturbations devrait permettre de conclure sur le mécanisme le plus sensible.

Remerciements. - Je tiens à remercier le Professeur W. P. Allis pour ses critiques pertinentes et pour son aide dans la préparation du manuscrit.

\section{Bibliographie}

[1] Davis, W. D., Vanderslice, T. A., Ion Energies at the cathode of a glow discharge, Phys. Rev. 131 (1963) 219.

[2] Merr, F. J., Biondi, M. A., Electron temperature dependence of electron ion recombination in argon, Phys. Rev. 176 (1968) 322.

[3] Brown, C., Basic Data of Plasma Physics (John Wiley, New York) 1959.

[4] BLoyet, J., Perturbation de la lueur négative d'une décharge luminescente par une onde hyperfréquence, L. P. 105, Laboratoire de Physique des Plasmas, Orsay.

[5] Solntsev, G. S., Orlov, A. I., Gerasimov, N., Influence d'une onde centimétrique sur les caractéristiques de la lueur négative d'une décharge luminescente dans He, Zh. Tekh. Fiz. 38 (1968) 893.

[6] O'MALLEY, T. F., Extrapolation of electron rare gas atom cross sections to zero energy, Phys. Rev. 130 (1963) 1020.

[7] Hамм, J. J., Technical Note $\mathrm{n}^{\circ}$ 2, Laboratoire de Physique des Plasmas, Orsay.
[8] Kantorek, P., Etude de la détection des ondes centimétriques à l'aide d'une décharge lumineuse, Czech. $J$. Phys. B 17 (1967).

[9] STERN, W., 8th International Conference on phenomena in ionized gases Panel 3-1-2 (1967).

[10] Cellarius, C. J., Dicks, L. A., TuRner, R., Determination of the densities and temperatures of low energy electrons groups in a hollow cathode discharge, $Z$. Phys. 231 (1970) 119.

[11] SeVERIN, P. J. W., Interaction des ondes centimétriques avec la chute cathodique et la lueur négative dans une décharge luminescente, Philips Research Reports, suppl. 2 (1965).

[12] Badareu, E., Popescu, T., Gaz ionisés. Décharges électriques dans les gaz (Dunod) 1969.

[13] Pringle, D. H., Farvis, W. E. J., Screened probe measurements in the helium negative glow, Proc. R. Soc. B 68 (1965) 836. 